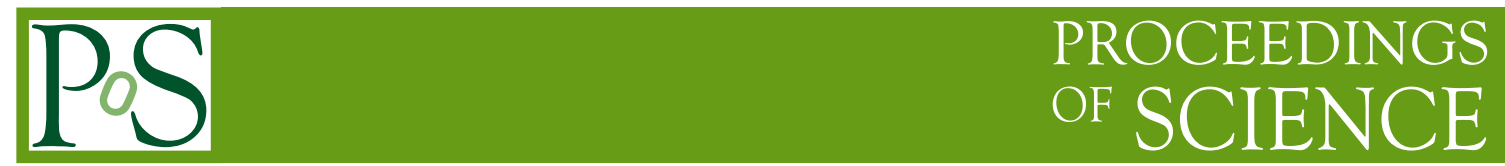

\title{
Constraints on new physics from the LHC
}

\section{Slava Krutelyov*i}

$U C S B$ - Texas A\&M

E-mail: vyacheslav.krutelyovecern.ch

\begin{abstract}
Highlights of searches for physics beyond the standard model are presented, covering work done at the LHC experiments, including ATLAS, CMS, and LHCb. The summary is focused primarily on results not covered in the rest of the program of the conference, with one exception of a search for rare $B_{s}^{0} \rightarrow \mu \mu$ decay. Following a brief overview of searches in supersymmetric and other exotic new physics scenarios, more details are given for searches probing supersymmetric scenarios. Naturalness considerations in supersymmetry bring forward topologies with multiple top and bottom quarks in the final states with rates in reach of the experiments. No evidence of new physics has been found so far.
\end{abstract}

14th International Conference on B-Physics at Hadron Machines April 8-12, 2013

Bologna, Italy

\footnotetext{
* Speaker.

${ }^{\dagger}$ For ATLAS, CMS, and LHCb collaborations.
} 


\section{Introduction}

The standard model (SM) of particle physics has been very successful in describing experimental data in a broad range of measurements [1]. The degree of agreement is still ever-improving across different fields, naming just some, in the heavy flavor physics [2], in the SM measurements at the LHC [3, 4], and in the precision electroweak observables [5]. The latest major milestone in the SM confirmation was the discovery of the Higgs boson [6, 7]. However, there is overwhelming experimental evidence, indicating that the SM is an effective theory and extensions to it are necessary to describe the neutrino mass and oscillation, the dark matter, origins of baryogenesis and necessary sources of $\mathrm{CP}$ violation, and, eventually, to incorporate gravity in the full picture. Combined with theoretical considerations, aiming, in particular, to address the hierarchy problem with diverging Higgs mass corrections, there is a broad range of theories beyond the SM (BSM).

The LHC experiments are currently at the energy and the intensity frontier for BSM searches. The ATLAS, CMS, and LHCb experiments have a broad program of BSM searches. While the $\mathrm{LHCb}$ is primarily focusing on the heavy flavor physics topics, the ATLAS and CMS lead the field of searches for the direct production of new heavy states, as reflected in publications $[8,9,10]$.

After a brief description of the experiments, this write up has details of a search-turn-measurement case in the $B_{s}^{0} \rightarrow \mu \mu$ process, followed by a review of selected topics in BSM searches by ATLAS and CMS. This report corresponds to the material available during the conference presentation on April 8, 2013. Updates can be found in preliminary or published documents posted by the experiments since then.

\section{Experiments}

The LHC is designed to deliver proton-proton collisions at a center-of-mass energy of $14 \mathrm{TeV}$ at a luminosity of $10^{34} \mathrm{~cm}^{-2} \mathrm{~s}^{-1}$ [11]. The LHC has completed in late 2012 its first major run in pp collisions at $8 \mathrm{TeV}$. The instantaneous luminosity has exceeded $7.5 \times 10^{33} \mathrm{~cm}^{-2} \mathrm{~s}^{-1}$, very close to the design parameters. The amount of data delivered to the experiments is equivalent of about $25 \mathrm{fb}^{-1}$ for the ATLAS and CMS, and about $3 \mathrm{fb}^{-1}$ for the LHCb, counting both the $8 \mathrm{TeV}$ running period in 2012 and the $7 \mathrm{TeV}$ one in 2011 [12]. A large fraction of this data has been analyzed by the experiments already in early 2013, as can be seen later in this report.

The ATLAS and CMS experiments operate general purpose hadron collider detectors [13, 14]. Coverage in an angle with respect to the beam line, $\theta$, or, equivalently, a pseudorapidity $\eta \equiv$ $-\ln [\tan (\theta / 2)]$, extends to $|\eta| \lesssim 5$. Each detector has a precision charged particle vertex and tracking detectors immersed in a solenoidal magnetic field and extending to $|\eta| \lesssim 2.5$, followed by finely segmented calorimetry with coverage up to $|\eta| \lesssim 5$ including forward calorimetry. Muon detectors are located outside the calorimeters in a region of $|\eta| \lesssim 2.5$. This coverage allows to efficiently measure a transverse momentum imbalance, $E_{\mathrm{T}}^{\mathrm{miss}}$, an indicator of neutrinos or other neutral particles escaping the detector, crucial elements in many BSM scenarios.

The LHCb is designed to be effective to collect final state particles from heavy flavor hadrons [15]. The detector is asymmetric, covering a range of $2 \lesssim \eta \lesssim 5$ and $2 \pi$ in azimuth on one side of the collision point. Similar detector features are present, as in the ATLAS and CMS. More instrumentation is present to separate muons from kaons and pions, essential for the heavy flavor physics. 


\section{Search for $B_{s}^{0} \rightarrow \mu \mu$ at $\mathbf{L H C b}$}

A rare decay $B_{s}^{0} \rightarrow \mu \mu$ is a flavor-changing neutral current process, strongly suppressed in the SM. Its rate is additionally reduced due to the helicity suppression. The expected branching fraction in the $\mathrm{SM}$ is $(3.23 \pm 0.27) \times 10^{-9}[16]$. This value can increase due to $b$-mixing by as much as about $9 \%$ after selecting decays with a significant life time. A similar mode $B^{0} \rightarrow \mu \mu$ has a rate additionally suppressed by $\left|V_{t d} / V_{t s}\right|^{2}$ with an expected branching fraction of $(1.07 \pm 0.10) \times 10^{-10}$. Significant enhancements are possible for in various BSM models. Some of the biggest theoretical interest for this mode has been for supersymmetric (SUSY) scenarios [17].

Searches for the $B_{s}^{0} \rightarrow \mu \mu$ decay have been a significant part of the physics program in many experiments. The Tevatron experiments $[18,19]$ were leading in the sensitivity until recently. Currently the LHC experiments are in the lead, with results reported by ATLAS [20], CMS [21], and $\mathrm{LHCb}$ [22]. A result from $\mathrm{LHCb}$, having the best observed sensitivity, is detailed below.

The analysis is performed by LHCb using $2 \mathrm{fb}^{-1}$ of data. Two normalization modes are used in this case: $B^{+} \rightarrow J / \psi K^{+}$, with $J / \psi \rightarrow \mu \mu$, and $B^{0} \rightarrow K^{+} \pi^{-}$(the charge conjugate states are implied). The signal selections require a well measured decay vertex displaced from a primary vertex with its momentum pointing back to the primary, consistent with b-hadron production with just two tracks in the decay vertex. The dimuon candidate should be isolated, not have a large number of charged particles in the candidate direction, suppressing gluon splitting and other backgrounds. The two muon candidates should pass high quality identification requirements suppressing misidentified hadrons. A two-stage multivariate selection, based on boosted decision trees, is applied to the $B_{(s)}^{0} \rightarrow \mu \mu$ candidates. A cut on the first multivariate discriminant removes $80 \%$ of the background while retaining $92 \%$ of signal. The output of the second multivariate discriminant, called BDT, and the dimuon invariant mass are used to classify the selected candidates. The candidates from the normalization modes are selected consistent with their decay final states. The multivariate selection is validated using the normalization $B^{0} \rightarrow K^{+} \pi^{-}$candidates as well as other similar two-hadron final states.

There are two sources of backgrounds: a combinatorial, from a random combination tracks, and a peaking, from fully or partially reconstructed decays of one $B$-hadron. Peaking backgrounds are constrained to known branching fractions and muon misidentification rates (measured in data). A mass shape fit is used to determine the backgronds using sidebands in a range of 4.9-6.0 GeV, excluding the signal regions $\pm 60 \mathrm{MeV}$ from $B_{(s)}^{0}$. In the $B_{s}^{0}$ signal window, after $B D T>0.8$, which gives about $70 \%$ of the sensitivity, the combinatorial backgrounds dominate: approximately 4.7 signal events are expected, for approximately 1.3 backgrounds and 5 events observed in the combined dataset (see the publication for details). In the $B^{0}$ signal window the two types of backgrounds are similar: approximately 0.6 signal events are expected with an additional feed down from $B_{s}^{0}$ signal of 0.8 , and a total background of 2.2, compared to 7 observed events.

The final results are extracted in a multivariate likelihood fit, performed in bins of BDT and invariant mass. The observed $B_{s}^{0}$ signal contribution has a statistical significance of $3.5 \sigma$ over backgrounds alone. The branching fraction obtained from the fit is $\left(3.2_{-1.2}^{+1.4} \mathrm{stat}_{-0.3}^{+0.5} \mathrm{syst}\right) \times 10^{-9}$, consistent with the SM. The upper limit on the branching fraction of the $B^{0} \rightarrow \mu \mu$ is found to be $9.4 \times 10^{-10}$ at $95 \%$ confidence level. With the current experimental sensitivity to $B_{s}^{0} \rightarrow \mu \mu$, this decay mode moves from the field of searches to the one of the SM measurements. Improved 
precision will increase the sensitivity to new physics.

\section{Birds-eye view of the searches}

Considering the searches in high-mass final states, both ATLAS and CMS experiments have a rich program covering BSM signatures, accounting for close to a half of their publications $[8,9]$. No evidence of BSM contributions have been seen so far and upper limits are reported. Summaries of the limits can be found online [23, 24, 25]. A cursory view shows that the limits for the same or similar scenarios are fairly close between the two experiments.

Depending on a type or a size of a coupling leading in a given BSM scenario, the limits extend to different masses of intermediate state resonances. For contact interactions the reach is up to $10 \mathrm{TeV}$, about $3 \mathrm{TeV}$ for states produced with strong coupling, and in a range of 1 to $2 \mathrm{TeV}$ for weakly interacting resonances. Sensitivity to final states which have to be produced in pairs due to some symmetry is naturally reduced.

\section{SUSY searches}

Supersymmetric extensions of the SM offer rather attractive scenarios addressing a range of theoretical issues, specifically, the hierarchy problem, they also offer a possibility of unification of interactions. Assuming R-parity conservation, the lightest supersymmetric particle (LSP) is a candidate for the dark matter. The supersymmetry has to be broken at some higher scale to match the (non)observation of the new SUSY particles. The phenomenology of the SUSY breaking is rather rich, a detailed review can be found elsewhere [1]. A SUSY scenario with a minimal Higgs sector having two Higgs doublets is the minimal supersymmetric standard model, MSSM. The lightest Higgs boson is SM-like. The value of its mass interconnects strongly possible (theoretically viable) ranges of masses of a gluino and the third generation superpartners.

Without an alternative protection of the Higgs mass, top squarks (stops) and gluinos are expected to be naturally at the TeV scale. Tensions arising from the Higgs boson mass being about $125 \mathrm{GeV}$ and improving limits at the LHC have prompted additional scrutiny of the concept of naturalness [26]. The naturalness now has a particularly high interest theoretically, to narrow down a range of less fine-tuned scenarios, and also experimentally, as a range of the theoretical scenarios suggest the stops and gluinos have to have masses in the LHC reach.

Superpartners of the Higgs bosons and of the SM electroweak bosons appear mixed in the MSSM in the mass eigenstates. Having an electroweak group charge, they are collectively called electroweakinos. Lower production cross sections imply lower experimental reach at the LHC in the electroweakino mass, compared to the strongly coupled gluino and squarks [27].

Complete SUSY scenarios provide concrete predictions of the spectrum of SUSY particles and their couplings. The phase space, however, is fairly large. Experimentally it is more convenient to work with simplified model scenarios (SMS) considering only a limited set of SUSY particles with fixed decay channels. This allows to probe a more complete phase space for this limited setup. 


\subsection{Electroweakino production in multilepton final states}

Electroweakino decays naturally have $W$ or $Z$ bosons in the chains and, subsequently, multiple leptons in the final states. ATLAS and CMS have investigated a chargino-neutralino production in a final state with three leptons and $E_{\mathrm{T}}^{\text {miss }}[28,29]$ as well as with four or more leptons [30]. In one SMS the leptons are from leptonic W/Z-boson decays, where the chargino (neutralino) decays to a W (Z) boson and an LSP. In the other SMS the electroweakinos decay via a slepton or sneutrino, yielding three leptons in the final state. These two SMS cover resonant and non-resonant sameflavor lepton pair production (on- $\mathrm{Z}$ and off-Z). An analysis using the full dataset at $8 \mathrm{TeV}$ reported by ATLAS in the three lepton final state is summarized briefly below.

The event selection requires three leptons with the lowest transverse momentum $p_{T}>10 \mathrm{GeV}$ and others as low as $14 \mathrm{GeV}$, depending on a trigger. These leptons are required to be isolated and come from the collision vertex, as expected for leptons from $\mathrm{W}$ or $\mathrm{Z}$ bosons, suppressing events with jets misidentified as leptons. A top-quark pair $(t \bar{t})$ production is suppressed by a veto of identified b-quark jets (b-tagged jets). A mass of a same-flavor oppositely charged (OSSF) lepton pair is used to separate the events into on- $\mathrm{Z}$ (within $10 \mathrm{GeV}$ of the $\mathrm{Z}$ mass) and off- $\mathrm{Z}$ categories.

The signal is searched in six categories, using the mass of the OSSF lepton pair, $E_{\mathrm{T}}^{\text {miss }}$, and the transverse mass $m_{T} \equiv \sqrt{2 \cdot E_{\mathrm{T}}^{\text {miss }} p_{T, \ell 3}\left(1-\cos \Delta \phi\left(\ell 3, E_{\mathrm{T}}^{\text {miss }}\right)\right)}$ using the third (not the OSSF) lepton. Three on- $\mathrm{Z}$ categories include two with a moderate $E_{\mathrm{T}}^{\text {miss }}(75-120 \mathrm{GeV})$, separated by $m_{T}$ into regions below and above $110 \mathrm{GeV}$; the third region has $E_{\mathrm{T}}^{\text {miss }}>120 \mathrm{GeV}$ and $m_{T}>110 \mathrm{GeV}$. Three off- $Z$ categories are sensitive to differences in masses between the intermediate and final state electroweakinos: the low mass splitting region has the OSSF mass below $60 \mathrm{GeV}$ and $E_{\mathrm{T}}^{\text {miss }}$ above $50 \mathrm{GeV}$; the intermediate splitting region has the OSSF mass between 60 and $81.2 \mathrm{GeV}$ with $E_{\mathrm{T}}^{\text {miss }}$ above $75 \mathrm{GeV}$; the high mass splitting region, excluded from the two above, has $m_{T}>110 \mathrm{GeV}$ and $p_{T, \ell 3}>30 \mathrm{GeV}$.

The dominant background contribution is irreducible, with all three leptons from $\mathrm{W}$ and $\mathrm{Z}$ bosons (WZ is the largest). Events with at least one misidentified lepton are less than $30 \%$. Expected and observed event yields are consistent with the SM alone. In the most restrictive signal selection regions 5 events are observed for $4.4 \pm 1.8$ backgrounds expected off-Z, and 6 observed for $6.3 \pm 1.5$ backgrounds expected on-Z. Upper limits for the two SMS are illustrated in Fig. 1.

\subsection{Gluino pair production via third generation superpartners}

A gluino pair production can be followed by decays primarily via a stop, since the stops can be the lightest among the squarks as suggested by naturalness. The stop can subsequently decay to a top quark and an LSP. The final state then contains two LSPs and four top quarks subsequently giving four Ws and b-quarks. This signature is very rich experimentally, it can be probed separately in events differing by the number of leptons; each case has substantially different SM backgrounds. This scenario has been probed by both ATLAS [31, 32, 33] and CMS [34, 35, 36, 37]. A result of a search in events with one lepton and multiple b-jets reported by CMS [35] is summarized here.

The event selection captures features of the signal: there should be one isolated lepton (electron or muon) with $p_{T, \ell}>20 \mathrm{GeV}$, at least six jets each with $p_{T}>40 \mathrm{GeV}$ with a sum of jet momenta $H_{T}>500 \mathrm{GeV}$. The sensitivity to the signal is enhanced by applying more strict requirements on $H_{T}$, a number of b-tagged jets, and $E_{\mathrm{T}}^{\text {miss }}$ or $S_{T} \equiv E_{\mathrm{T}}^{\text {miss }}+p_{T, \ell}$. The primary background is a $t \bar{t}$ 

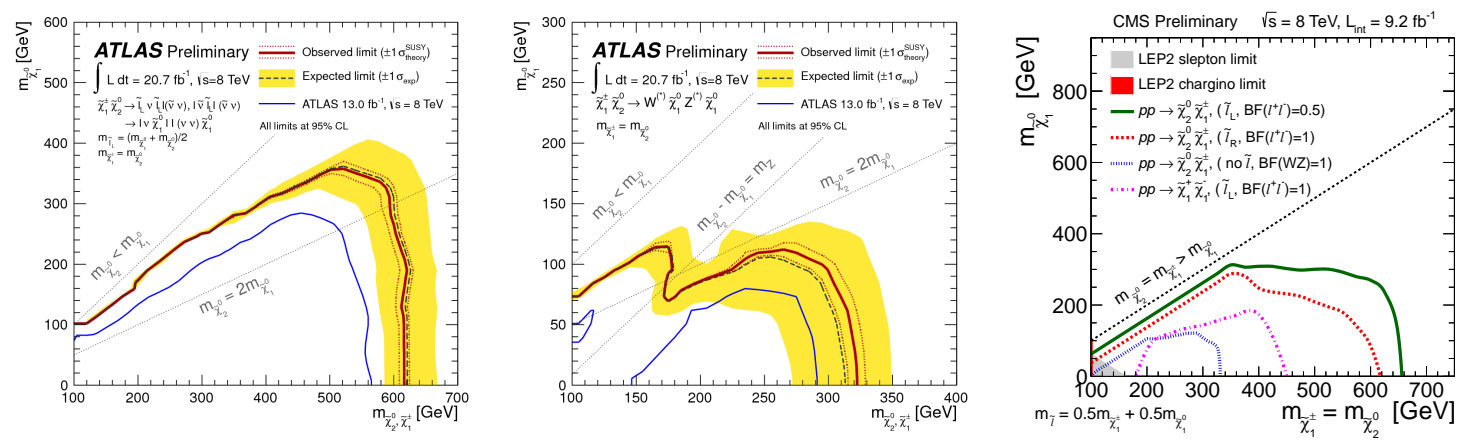

Figure 1: Constraints on chargino-neutralino production SMS with three leptons in the final state: produced via sleptons, mostly off- $Z$ on the left; produced via gauge bosons, mostly on- $Z$ in the center; compared to the CMS result on the right.

production. Two methods are applied in the analysis relying on features of the expected signal: one uses $E_{\mathrm{T}}^{\mathrm{miss}}$ and the other uses $S_{T}$.

The first method uses the lepton $p_{T, \ell}$ spectrum to predict the background $E_{\mathrm{T}}^{\text {miss }}$ distribution. This works approximately for the $t \bar{t}$, since the lepton and neutrino spectra are related. Corrections are applied to account for the polarization of the $\mathrm{W}$, the experimental $E_{\mathrm{T}}^{\mathrm{miss}}$ resolution, and contribution from $t \bar{t}$ decays with two leptons or with taus. Since the method relies on the $E_{\mathrm{T}}^{\text {miss }}$ distribution properties, the signal selections rely on $E_{\mathrm{T}}^{\text {miss }}$. The dominant background comes from events with only one genuine lepton in the top-quark pair production. In one of the most sensitive regions with $E_{\mathrm{T}}^{\text {miss }}>450 \mathrm{GeV}, H_{T}>750 \mathrm{GeV}$, and at least two b-tagged jets there is one observed event for an expectation of $0.7_{-0.3}^{+2.5}$ background and $2.7 \pm 0.3$ signal events with a gluino mass of $1.15 \mathrm{TeV}$.

The second method uses a fact that in the SM events with only one W decaying leptonically the $\mathrm{W}$ momentum corresponds to a vector sum of the lepton and $E_{\mathrm{T}}^{\text {miss }}$ matching the neutrino. If this $\mathrm{W}$ has a high momentum, an angle $\Delta \phi(W, \ell)$ between the $\mathrm{W}$ and the lepton directions is small. For the signal the presence of LSPs removes the correlation. A requirement of $\Delta \phi(W, \ell)>1$ rejects most of the genuine single-lepton background. Events with $\Delta \phi(W, \ell)<1$ are dominated by backgrounds and are used to normalize the backgrounds prediction. Signal sensitivity is improved by analysis in bins of $S_{T}$ from 250 to 350 , to $450 \mathrm{GeV}$ and above. In one of the most sensitive regions with $S_{T}>450 \mathrm{GeV}$ and at least three b-tagged jets no events are observed for a background of $0.0 \pm 0.3$ expected, compared to approximately 3.6 signal expected for a gluino mass of $1.15 \mathrm{TeV}$.

Background expectations are found to be in agreement with observed data. Constraints on the signal production are shown in Fig. 2. The gluino masses are excluded below 1.3 TeV.

\subsection{Top squark pair production}

From the naturalness considerations the stop can be the lightest strongly coupled SUSY particle. With all other quark and gluon superpartners possibly out of experimental reach, the stop pair production becomes the only available channel in the strong coupling production of the SUSY particles. The simplest scenario is for the produced stop to decay directly to an LSP and a top quark. The final state is then a $t \bar{t}$ with a pair of LSPs. A similar final state can arise if the stop decays to a chargino and a b-quark with the chargino subsequently decaying to a W boson and an LSP. In 

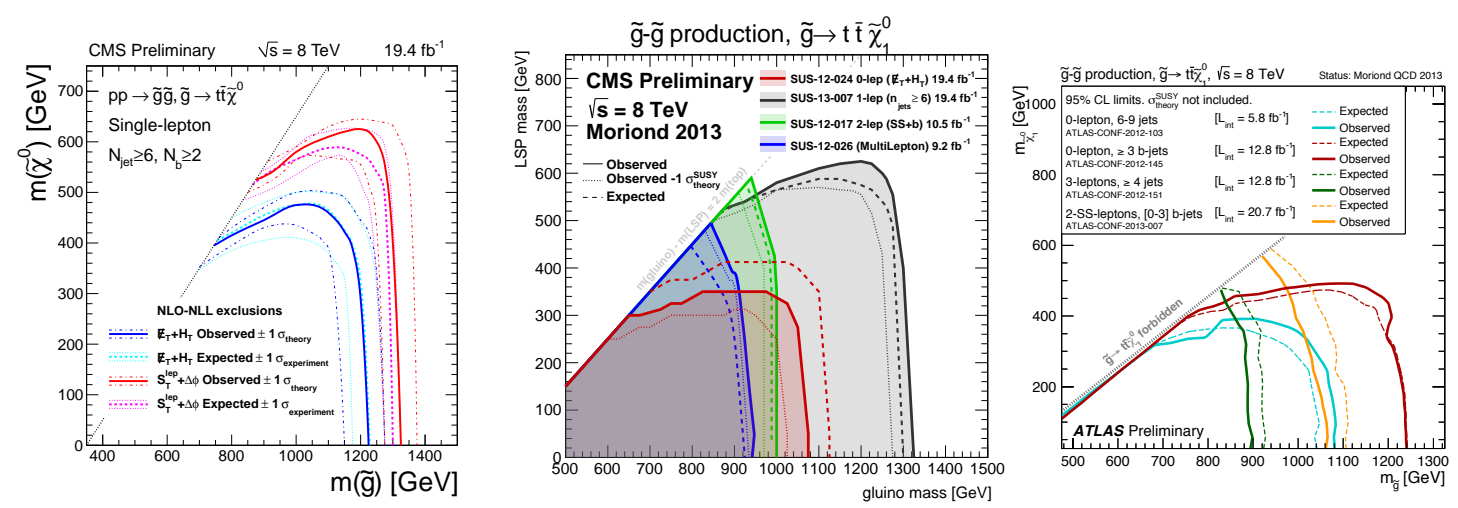

Figure 2: Constraints on gluino pair production with four top quarks and two LSPs in the final state: using events with one lepton (left), comparing with other searches from CMS (center), and comparing with similar searches at ATLAS (right).

this case there is no resonance in a combination of the b-quark and the W. Results of searches for the stop pair production have been recently reported by the ATLAS and CMS experiments for the $8 \mathrm{TeV}$ datasets $[38,39,40]$. A summary of a result reported by ATLAS using a final state with one lepton, jets, and $E_{\mathrm{T}}^{\mathrm{miss}}$ is provided here.

The analysis selects events with one lepton (electron or muon) with $p_{T}>25 \mathrm{GeV}$, at least four jets with $p_{T}$ above $80,60,40$, and $25 \mathrm{GeV}$, with at least one of these jets b-tagged. For all signal selections $E_{\mathrm{T}}^{\mathrm{miss}}$ is above $100 \mathrm{GeV}$. At this level, most of the backgrounds are from a $t \bar{t}$ production and a W-boson production with jets.

Sensitivity to the two stop pair decay scenarios is refined further. Three signal regions are defined for the stop decaying directly to a top quark and an LSP and three regions are defined for the case of the stop decaying via a chargino. The main difference for the former is the requirement that there should be three jets kinematically consistent with a top quark decay. Two jets with the highest momenta should be separated from the $E_{\mathrm{T}}^{\text {miss }}$ direction essentially for all cases to suppress mismeasurements. The transverse mass variable $m_{T}$ is above at least $120 \mathrm{GeV}$ for decays via a chargino with tighter requirements for the other case - this requirement suppresses most of the SM backgrounds including only one $\mathrm{W}$ boson decaying leptonically. Significant contributions from $t \bar{t}$ with both Ws decaying leptonically and one lepton undetected are suppressed with variables $\left(m_{T 2}\right.$ variables) aiming to disentangle contributions of the two neutrinos to the $E_{\mathrm{T}}^{\text {miss }}$. These $m_{T 2}$ variables have distributions terminating at the top quark mass for the SM $t \bar{t}$ production. In one of the signal regions aiming at top quarks and LSPs the $m_{T}$ and $E_{\mathrm{T}}^{\text {miss }}$ shapes are used. In all other cases simple event counts are used. As an illustration, in the most restrictive signal region probing decays with charginos there are 6 observed events for $7 \pm 3$ background events expected roughly split among $t \bar{t}, \mathrm{~W}+\mathrm{jets}$ and single top quark production contributions. Similarly, the most restrictive selections targeting the signal with stops decaying to tops and neutralinos has 7 observed events compared to $5 \pm 2$ expected backgrounds with about a third each from $t \bar{t}$ and $\mathrm{W}+$ jets with the remainder split between single top production and $t \bar{t} Z$ and $t \bar{t} W$ production.

A summary of constraints placed on the stop pair production is given in Fig. 3. The exclusion extends up to 650 to $700 \mathrm{GeV}$. 

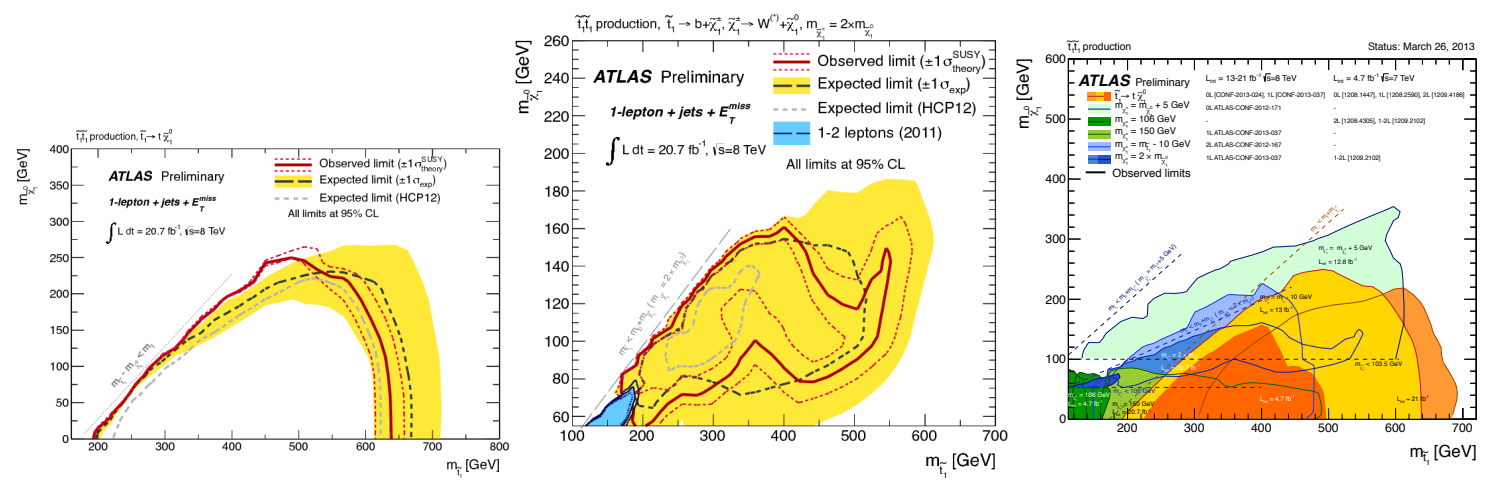

Figure 3: Constraints on stop pair production: using events with one lepton for decays to top quarks (left), events with one lepton for decays via a chargino (center), comparing with other searches by ATLAS (right).

\subsection{Summary}

The physics program of searches for new physics beyond the standard model at the LHC has been very successful in constraining various BSM scenarios. No evidence of new physics signal has been observed so far. After completing analysis of the data delivered by the LHC up to the end of 2012 the next crucial steps are to prepare for data analysis at a higher energy when the LHC returns back online in 2015 .

\section{References}

[1] J. Beringer et al. (Particle Data Group), Review of Particle Physics (RPP), Phys. Rev. D 86 (2012) 010001.

[2] Y. Amhis et al. (Heavy Flavor Averaging Group), Averages of B-Hadron, C-Hadron, and tau-lepton properties as of early 2012, arXiv:1207.1158.

[3] ATLAS summary of Standard Model analyses https://twiki.cern.ch/twiki/bin/view/ AtlasPublic/StandardModelPublicResults.

[4] CMS summary of Standard Model analyses https://twiki.cern.ch/twiki/bin/view/CMSPublic/PhysicsResultsSMP.

[5] M. Baak and R. Kogler, The global electroweak Standard Model fit after the Higgs discovery, arXiv:1306.0571.

[6] ATLAS Collaboration, Observation of a new particle in the search for the Standard Model Higgs boson with the ATLAS detector at the LHC, Phys. Lett. B 716 (2012) 1 [arXiv:1207.7214 [hep-ex]].

[7] CMS Collaboration, Observation of a new boson at a mass of $125 \mathrm{GeV}$ with the CMS experiment at the LHC, Phys. Lett. B $\mathbf{7 1 6}$ (2012) 30 [arXiv:1207.7235 [hep-ex]].

[8] ATLAS Papers https://cds.cern.ch/collection/ATLAS\%20Papers?ln=en.

[9] CMS Papers https://cds.cern.ch/collection/CMS\%20Papers?ln=en.

[10] LHCb Papers https://cds.cern.ch/collection/LHCb\%20Papers?In=en.

[11] L. Evans and P. Bryant, LHC Machine, JINST 3 (2008) S08001. 
[12] LHC Performance and Statistics

https://lhc-statistics.web.cern.ch/LHC-Statistics/.

[13] ATLAS Collaboration, The ATLAS Experiment at the CERN Large Hadron Collider, JINST 3 (2008) S08003.

[14] CMS Collaboration, The CMS experiment at the CERN LHC, JINST 3 (2008) S08004.

[15] LHCb Collaboration, The LHCb Detector at the LHC, JINST 3 (2008) S08005.

[16] A. J. Buras, J. Girrbach, D. Guadagnoli and G. Isidori, On the Standard Model prediction for $B R\left(B_{s, d}^{0} \rightarrow \mu^{+} \mu^{-}\right)$, Eur. Phys. J. C 72 (2012) 2172 [arXiv:1208.0934 [hep-ph]].

[17] K. S. Babu and C. F. Kolda, Higgs mediated $B^{0} \rightarrow \mu^{+} \mu^{-}$in minimal supersymmetry, Phys. Rev. Lett. 84 (2000) 228 [hep-ph/9909476].

[18] T. Aaltonen et al. [CDF Collaboration], Search for $B_{s}^{0} \rightarrow \mu^{+} \mu^{-}$and $B^{0} \rightarrow \mu^{+} \mu^{-}$decays with the full CDF Run II data set, Phys. Rev. D 87 (2013) 072003 [arXiv:1301.7048 [hep-ex]].

[19] V. M. Abazov et al. [D0 Collaboration], Search for the rare decay $B_{s}^{0} \rightarrow \mu \mu$, Phys. Rev. D 87 (2013) 072006, [arXiv:1301.4507 [hep-ex]].

[20] ATLAS Collaboration, Search for the decay $B_{s}^{0} \rightarrow \mu \mu$ with the ATLAS detector, Phys. Lett. $B \mathbf{7 1 3}$ (2012) 387 [arXiv:1204.0735 [hep-ex]].

[21] CMS Collaboration, Search for $B_{s}^{0} \rightarrow \mu^{+} \mu^{-}$and $B^{0} \rightarrow \mu^{+} \mu^{-}$decays, JHEP 1204 (2012) 033 [arXiv:1203.3976 [hep-ex]].

[22] LHCb Collaboration, First Evidence for the Decay $B_{s}^{0} \rightarrow \mu^{+} \mu^{-}$, Phys. Rev. Lett. 110 (2013) 021801 [arXiv:1211.2674 [hep-ex]].

[23] ATLAS Physics Summary Plots https:

//twiki.cern.ch/twiki/bin/view/AtlasPublic/CombinedSummaryPlots

[24] CMS Exotica Public Physics Results

https://twiki.cern.ch/twiki/bin/view/CMSPublic/PhysicsResultsEXO.

[25] CMS Supersymmetry Physics Results

https://twiki.cern.ch/twiki/bin/view/CMSPublic/PhysicsResultsSus.

[26] J. L. Feng, Naturalness and the Status of Supersymmetry, arXiv:1302.6587 [hep-ph].

[27] W. Beenakker, M. Klasen, M. Kramer, T. Plehn, M. Spira and P. M. Zerwas, The Production of charginos / neutralinos and sleptons at hadron colliders, Phys. Rev. Lett. 83 (1999) 3780 [Erratum-ibid. 100 (2008) 029901] [hep-ph/9906298]; plots available at http://www.thphys. uni-heidelberg.de/ plehn.

[28] ATLAS Collaboration, Search for direct production of charginos and neutralinos in events with three leptons and missing transverse momentum in $21 \mathrm{fb}^{-1}$ of pp collisions at $\sqrt{\mathrm{s}}=8 \mathrm{TeV}$ with the ATLAS detector, ATLAS-CONF-2013-035.

[29] CMS Collaboration, Search for direct EWK production of SUSY particles in multilepton modes with $8 \mathrm{TeV}$ data, CMS-PAS-SUS-12-022.

[30] ATLAS Collaboration, Search for supersymmetry in events with four or more leptons in $21 \mathrm{fb}^{-1}$ of pp collisions at $\sqrt{s}=8 \mathrm{TeV}$ with the ATLAS detector, ATLAS-CONF-2013-036.

[31] ATLAS Collaboration, Search for gluino pair production in final states with missing transverse momentum and at least three b-jets using $12.8 \mathrm{fb}^{-1}$ of pp collisions at $\sqrt{s}=8 \mathrm{TeV}$ with the ATLAS Detector, ATLAS-CONF-2012-145. 
[32] ATLAS Collaboration, Search for strongly produced superpartners in final states with two same sign leptons with the ATLAS detector using $21 \mathrm{fb}^{-1}$ of proton-proton collisions at $\sqrt{\mathrm{s}}=8 \mathrm{TeV}$, ATLAS-CONF-2013-007.

[33] ATLAS Collaboration, Search for supersymmetry using events with three leptons, multiple jets, and missing transverse momentum in $13.0 \mathrm{fb}^{-1}$ of pp collisions with the ATLAS detector at $\sqrt{\mathrm{s}}=8 \mathrm{TeV}$, ATLAS-CONF-2012-151.

[34] CMS Collaboration, Search for gluino mediated bottom- and top-squark production in multijet final states in pp collisions at $8 \mathrm{TeV}$, arXiv:1305.2390 [hep-ex].

[35] CMS Collaboration, Search for Supersymmetry in pp collisions at $8 \mathrm{TeV}$ in events with a single lepton, multiple jets and b-tags, CMS-PAS-SUS-13-007.

[36] CMS Collaboration, Search for new physics in events with same-sign dileptons and b jets in pp collisions at $\sqrt{s}=8 \mathrm{TeV}, \mathrm{JHEP} 1303$ (2013) 037 [arXiv:1212.6194 [hep-ex]].

[37] CMS Collaboration, A Search for Anomalous Production of Events with three or more leptons using 9.2 $\mathrm{fb}^{-1}$, CMS-PAS-SUS-12-026.

[38] CMS Collaboration, Search for direct top squark pair production in events with a single isolated lepton, jets and missing transverse energy at $\sqrt{s}=8 \mathrm{TeV}$, CMS-PAS-SUS-12-023.

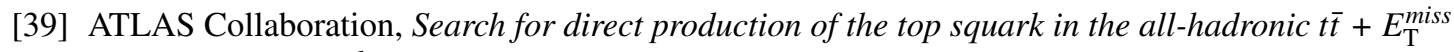
final state in $21 \mathrm{fb}^{-1}$ of pp collisions at $\sqrt{\mathrm{s}}=8 \mathrm{TeV}$ with the ATLAS detector, ATLAS-CONF-2013-024.

[40] ATLAS Collaboration, Search for direct top squark pair production in final states with one isolated lepton, jets, and missing transverse momentum in $\sqrt{s}=8 \mathrm{TeV}$ pp collisions using $21 \mathrm{fb}^{-1}$ of ATLAS data, ATLAS-CONF-2013-037. 